\title{
6. Miszellaneen
}

Dieses Kapitel befasst sich mit Briefen (oder Ausschnitten aus einem Brief), deren Autoren als >Augenzeugen pekten des Falls Hutten Stellung nehmen, indem sie schreiben, was sie beobachtet, erfahren oder erlebt haben: der Brief des Landsknechts Diego Ruiz de Vallejo, Ausschnitte aus einem längeren, in florentinischem Italienisch verfassten, als Brief markierten Reisebericht von Galeotto Cei und der Brief des Richters Juan Pérez de Tolosa, in dem er dem König seine Verhaftungsexpedition nach El Tocuyo schildert. Das Mandat Kaiser Karls V. wird als (indirekte) Antwort auf Tolosas Brief gelesen und deshalb im selben Unterkapitel behandelt.

Die Autoren der drei Briefe treten alle als Figuren im Kriminalprozess auf: Diego Ruiz de Vallejo als aussagender Zeuge (vgl. Manuskript aus dem AGI), Galeotto Cei als Unterzeichnender des Friedensvertrags zwischen Hutten und Carvajal und des Geständnisses von Carvajal am Fuße des Galgens (vgl. M: 507 und 543, dort als »Galeote They« verzeichnet) und schließlich Juan Pérez de Tolosa bekanntlich als Richter. Das Mandat (die real cédula) Kaiser Karls V. wird in Kapitel 6 im Zusammenhang mit dem Brief Tolosas näher betrachtet, weil sie inhaltlich aufeinander Bezug nehmen. Das kaiserlich-königliche Schreiben richtet sich allerdings nur indirekt an den Richter; direkter Adressat war der Consejo de Indias (Indienrat), der Richter Tolosa anweisen sollte, das Verfahren gegen die Schuldigen zu Ende zu führen, die an den Verbrechen gegen Hutten und Welser neben Carvajal beteiligt gewesen sein sollen.

In den drei Briefen konstituiert sich jeweils ein koloniales Subjekt, das je nach der spezifischen kolonialen Situation in unterschiedlicher Weise sinterpelliert< wird. Dabei tanzt der italienische Text buchstäblich aus der Reihe; sowohl Ceis Text selbst als auch die kritische Literatur zeigen Eigenheiten, die sich von den anderen deutlich unterscheiden. Zum Schluss kommt das Mandat Kaiser Karls V. zur Sprache, das eigene Fragen aufwirft. 


\section{Der Brief des Diego Ruiz de Vallejo}

Diego Ruiz de Vallejo, bei Eberhard Schmitt und Götz Simmer (1999: 88) als »Landsknecht « bezeichnet, war 1545 mit Carvajal nach El Tocuyo gezogen und lief $1546 \mathrm{zu}$ Huttens Truppe über (vgl. ebd.: 182). Mit seinem Brief an einen Unbekannten setzt er Philipp von Hutten ein Denkmal. Er schildert seinem Adressaten ausführlich die Geschehnisse um Philipp von Huttens und Bartholomäus Welsers Tötung und modelliert dabei gleichzeitig Philipps Figur als eine Art charismatische "Lichtgestalt« (Denzer 2005: 169), die, wie Denzer ausführt, von den späteren Chronisten aufgegriffen wurde. Huttens Figur hebe sich dort insgesamt stark von den übrigen »düsteren Protagonisten der Welserzeit« ab (ebd). Das >Monument< Philipp von Hutten, das sich aus dem Gesamt der Signifikationen der Texte ergibt, findet hier möglicherweise einen seiner Ankerpunkte.

Als Merkmal eines typisch >kolonialen Textes` rückt die textuelle Marginalisierung der »indios« ins Zentrum. Die >Kontaktzone zwischen Konquistadoren und Indigenen nimmt - im Vergleich mit Huttens Briefen - kaum mehr textuellen Raum ein. Diego Ruiz de Vallejo hat seinen Brief gut fünf Jahre nach dem letzten Brief von Philipp von Hutten verfasst; es scheint, als ob die Eroberung schon sehr weit fortgeschritten und der Widerstand minimiert worden seien. Die Kämpfe gegen Huttens »Indier« werden bei Vallejo durch Kämpfe zwischen den beiden verfeindeten Fraktionen der Konquistadoren ersetzt, die jeweils von Hutten beziehungsweise von Carvajal angeführt werden. Wie selbstverständlich reiht das Schreiben die »indios«, sofern sie überhaupt erwähnt werden, in das Tun der europäischen Konquistadoren ein. Der Sklave, der Vallejo nach seiner Darstellung das Leben gerettet hat, sowie »Perico« und »Magdalena«, die beiden »indios«, die Philipp von Hutten gedient hatten und die Carvajal entkommen sind, erscheinen weder als Bedrohung noch als unzuverlässige Kolonialisierte. Im Gegenteil: Sie spielen eine den Kolonialherren dienende Rolle. Andere namenlose »indios« erscheinen höchstens als virtuelle Gefahr: als Vallejo den Alarm, den Carvajals Überfall auf Huttens Lager auslöste, im ersten Moment des Schreckens als Überfall der »indios « missdeutete oder der >Stamm` der Omaguas, über dessen Gefährlichkeit Hutten sich gemäß Vallejos Darstellung nach der Rückkehr von seiner entrada informieren wollte. Wie schon von Philipp von Hutten befürchtet, öffnet sich der Graben nun vor allem zwischen den »Christen« selbst - den verfeindeten Fraktionen unter den Konquistadoren. Die Indigenen treten lediglich in ihrer Reaktion auf das Handeln der Konquistadoren textuell in Erscheinung. Da- 
mit macht der Text, wovon er spricht: Er spricht den Indigenen wortwörtlich keinen Platz für ein Eigenleben zu.

Diego Ruiz de Vallejo richtet seinen Brief vom 28. Juni 1546 an einen gewissen »muy noble señor « $\left(\mathrm{R}^{1}: 245\right)$, um diesen über die Geschehnisse in der Provinz zu unterrichten. Da er ihn nicht namentlich anspricht, wurde verschiedentlich über die Identität des Adressaten spekuliert (vgl. Haebler 1903: 338; Friede 1961: 414, zitiert nach Schmitt/Simmer 1999: 99); eine Frage, die, wie wir sehen werden, nicht abschließend geklärt wurde. Wer aber war der Schreiber des Briefes? Nach Götz Simmer war Ruiz de Vallejo ein »Landsknecht«, der vermutlich bereits an Hohermuths Entrada teilgenommen hatte, später Juan de Carvajal nach El Tocuyo gefolgt, dort aber zu Hutten übergelaufen ist (vgl. Simmer 1999b: 182f.). Dass er seinen Brief an einen »muy noble señor « richtet, legt nahe, dass er Zugang zur Hierarchie des bürokratischen Apparats hatte. Auch die Tatsache, dass er später »verschiedene Ämter [und] nach 1561 zum königlichen Rentmeister (Contador de la Real Hacienda) von Venezuela ernannt « (ebd.: 183) wurde, weist in dieselbe Richtung. Der Brief muss also als »kolonialer Text« im Sinne Kienings (vgl. 2003) gelesen werden, insofern er sich mit einem Ausschnitt der Eroberung beschäftigt. Vallejo versucht, diese zu organisieren, indem er Missstände anspricht und sich an einen Adressaten richtet, von dem er annimmt, dass dieser die Informationen benötigt, um dann angemessen handeln zu können. Als Zudiener der Macht zu gelten, könnte das Ziel des Schreibens gewesen sein, womit auch die Interpellation in Gang gesetzt worden sein dürfte.

Ruiz de Vallejo erschreibt sich eine Position als >Wissender<, als unverzichtbare Quelle wichtiger Informationen: Er kündigt seinem Adressaten in einer Art Vorspann seines Briefes an, ihm von den Geschehnissen im Land $\mathrm{zu}$ berichten, damit dieser wisse, was laufe (»para que sepa lo que pasa«, R: 245). Die Erzählwürdigkeit - oder besser: Erzählnotwendigkeit - schöpft Vallejo aus der Ungeheuerlichkeit der Vorkommnisse, die, so liest es sich zwischen den Zeilen, ein Handeln oder Eingreifen durch den Adressaten erfordern.

Anlass des Schreibens ist der von Ruiz de Vallejo beobachtete blutige Konflikt zwischen Philipp von Hutten und Juan de Carvajal; es wird über die Kanäle und Mechanismen des bürokratischen Apparats ventiliert und angetrieben. Vallejo setzt sein >Kapitak, bestehend aus Informationen aus erster Hand, so ein, als ob ihm eine potentielle Honorierung in Aussicht gestellt würde. Als berichtender Zeuge lässt er den Leser des Briefes unmittelbar an seinen Wahrnehmungen teilhaben; er berichtet, was er in den bedrohlichsten Momenten 
sieht, hört und denkt. Damit erschreibt er sich eine Stellung als exklusive Quelle der >Wahrheit über die berichteten Vorgänge.

Als Überläufer kennt Ruiz de Vallejo sowohl die Perspektive Carvajals als auch diejenige Huttens. Im Verlauf des Briefes ergreift er verstärkt Partei für Letzteren, ihn zur genannten >Lichtgestalt'formend. Carvajal hinderte Hutten bekanntlich an der Weiterreise nach Coro, wo Hutten dem König und dem damaligen Juez de Residencia, Juan de Frías, über die Ergebnisse seiner entrada Bericht erstatten wollte. Die Darstellung dieser Auseinandersetzung zeichnet sich aus durch den Einsatz direkter Reden, die den Figuren Hutten und Carvajal in den Mund gelegt werden und diese dadurch als >Lichtgestalt respektive als $>$ Bösewicht< charakterisieren.

Als Basis der Analyse liegt mir Arellano Morenos' (vgl. R) Transkription vor, die ich bei Unklarheiten ${ }^{2}$ mit dem Scan des siebenseitigen Manuskripts des AGI abgeglichen habe. Der Brief findet in deutscher Übersetzung Eingang in die Zusammenstellung der Dokumente bei Schmitt/Simmer (vgl. 1999: 99108). Er ist überschrieben mit folgendem Titel: »[T]reslado de vna carta que escribe Diego Ruyz de Vallejo de Coro a 28 de junio 1546 es lo siguiente « ${ }^{3}$ (R: 245), und endet abrupt, ohne den charakteristischen abschließenden Gruß, was darauf hinweist, dass der Schluss des Briefes verschollen ist.

Die Identität des Adressaten ist nicht geklärt. Dass nur der Präsident der Audiencia von Santo Domingo, ein gewisser licenciado Cerrato, in Frage komme, wie Schmitt/Simmer (vgl. 1999: 99) nahelegen, erscheint eher zweifelhaft. Denn dieser wird im letzten Satz namentlich genannt, und dies auch noch wenig schmeichelhaft: „toda la culpa desto [la muerte de Hutten; SG] no la echan en esta tierra a nadie sino es al señor licenciado cerrato presidente « ${ }^{4}(R$ : 251). Es ist schwer vorstellbar, dass der Verfasser des Briefes, Ruiz de Vallejo, seinen (noblen!) Adressaten so offen und direkt beschuldigen würde, für die blutigen Vorfälle verantwortlich zu sein. Überdies stimmt die grammatische Person nicht: Wäre Cerrato der Adressat, würde Vallejo diesen direkt mit usted ansprechen, wie er es im ersten Satz des Briefes auch tut. Interesse an den Vorkommnissen dürfte dagegen bei Personen aus dem Umfeld der Welser als Verwalter der Provinz bestanden haben. In diesem Sinne leuchtet der Vorschlag Konrad Haeblers (vgl. 1903: 338) eher ein, der - allerdings ohne weitere Begründung oder Belege - Bartholomäus Welser der Ältere, den Führer der Welser-Kompanie, zum Adressaten erklärt. Ruiz de Vallejo schrieb den Brief, nachdem er als Zeuge im Kriminalprozess gegen Carvajal Zeugnis abgelegt hatte. Dass der Richter, Juan Pérez de Tolosa, von Anfang an von der Schuld 
des Angeklagten ausging und dies auch öffentlich verkündete, floss mit Sicherheit in den Tenor des Briefes ein.

Der Erzähler kreiert einen Spannungsbogen von den ersten Meutereien gegen Bartholomäus Welser über die Scharmützel zwischen Carvajal und Hutten bis zu dessen Enthauptung und den mit diesem Verlust einhergehenden Schaden, den die Provinz genommen habe, denn Hutten habe große Pläne mit der Provinz gehabt: »[E]l capitan general traya gran noticia y tal que queria gastar 10 o 12 mil ducados para traer de castilla gente rropa y cauallos y ganados desa ysla y queria hacer tres pueblos « ${ }^{5}$ (R: 250). Der Schreiber deutet hier an, dass mit dem Tod Philipps von Hutten auch die Zukunft der Provinz Venezuela, vielleicht sogar ihre Zukunft unter der Statthalterschaft der Welser, untergehen könnte. Eine solche Lesart würde für einen Adressaten im Umkreis der Welser sprechen. Vallejo geht aber noch weiter, wenn er Hutten sogar als potentiellen Landesvater evoziert: Bis auf sechs Leute hätten ihn alle beweint, und solange sie in den Indias seien, würden sie ihn beweinen, "porque dizen que no a sido capitan general sino padre de todos « ${ }^{6}$ (R: 250f.).

Die Positionen sind klar: Vallejo ergreift Partei für Hutten. Sich selbst stellt er als loyalen Untergebenen des seinerseits dem König bzw. den Welsern treu ergebenen Hutten dar. Er preist seinen Brief als den einzig Wahren an, da er, im Gegensatz zu nicht näher genannten sanderen` Briefeschreibern, salles` selbst erlebt habe und somit als Augenzeuge auftreten kann (vgl. ebd.: 245). Er habe die Festnahme Huttens und Welsers beobachtet, sei daraufhin aber geflohen und gibt die Enthauptung der beiden aus indirekter Quelle, gemäß den Aussagen der beiden Sklaven wieder, die Philipp von Hutten gedient hatten und die ebenfalls entkommen sind.

Vallejo entwertet den Brief eines gewissen Pedro de Ávila, den dieser nur geschrieben habe, um ihn Carvajal zu zeigen, und der nicht die Wahrheit enthalte. Denn die Menschen pflegten unter »solchen« (»tales« R: 245) Gouverneuren wie Juan de Carvajal unterwürfig zu werden; so habe es auch Ávila nicht gewagt, die Wahrheit zu schreiben: »[E]sta subjecion tienen los hombres mas con tales governadores no osar escrevir verdad « ${ }^{7}$ (R: 245). Damit wird Carvajal einmal mehr als unwürdiger Machthaber dargestellt.

Nach der Ankündigung, über die Ereignisse im Land aus der Sicht eines direkt Beteiligten zu berichten, setzt die Erzählung aus allwissender Perspektive etwas unvermittelt an einem Punkt der Geschichte ein, an dem sich erste Entzweiungen zwischen den beiden Rivalen Hutten und Carvajal bereits abzeichnen. Der Einschnitt ist im Manuskript durch einen rechteckigen, nach 
rechts offenen Rahmen markiert. Ob diese Markierung vom selben Schreiber oder einem späteren Leser stammt, ist aus dem mir vorliegenden PDFDokument nicht ersichtlich.

Als erste handelnde Person wird nicht einer der Protagonisten Hutten oder Carvajal, sondern Pedro de Limpias eingeführt, der in Begleitung von sechs Männern im Lager Carvajals auftaucht: »Por el principio del mes de hebrero estando en el asiento que dizen del tocuyo juan de carvajal que es en barquisimeto llego una noche pedro de linpias adonde estava carvajal y hablo con juan de villegas $\aleph^{8}$ (R: 245). In den folgenden zehn Zeilen werden Zeit, Ort und fast alle Hauptpersonen eingeführt. Es häufen sich Namen von Beteiligten, was dem Schreiben eine beglaubigende Note aufdrückt. Für das Erzählen beziehungsweise das Nachvollziehen des Gelesenen sind sie jedenfalls nicht von Bedeutung; sie werden sich an späterer Stelle auch nicht wiederholen. Die Neuankömmlinge sind, wie sich im nächsten Satz herausstellt, Überläufer, die sich zuerst gegen Bartholomäus Welser erhoben hatten und jetzt von Huttens Seite zu Carvajal wechseln möchten.

Der Name Philipp von Hutten fällt erstmals in der nächsten Zeile, in der der Erzähler die Überläufer in indirekter Rede über ihre Erlebnisse während Huttens »jornada« (ebd.) berichten lässt. Das Verb perder (`verlieren`) wird dabei zu ihrem Markenzeichen: Der Spur Hernán Pérez de Quesadas folgend, hätten sie sich verirrt (»se avian perdido«, ebd.), in einer Sierra hätten sie alle ihre Pferde verloren bis auf acht (»en una sierra perdieron todos los cavallos que no escaparon sino ocho«, ebd.: 245f.). Die Teilnehmer der Hutten-entrada scheinen also dem Verderben sehr nahegekommen zu sein. Immer noch im Modus der indirekten Rede wird erzählt, dass Hutten Welser nach Coro vorausgeschickt habe, um über seine Reise zu berichten und Hilfe zu holen (vgl. ebd.: 246).

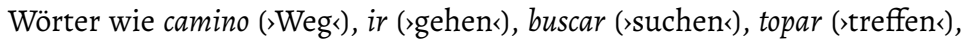
llegar (`ankommen`), enviar (`schicken`) prägen nun dem Schreiben Bewegung auf. Der Wechsel zwischen Nähe und Distanz spielt sich wie in einem Katzund-Maus-Spiel zwischen den verfeindeten Fraktionen der Konquistadoren ab. Der camino als Voraussetzung des Vorankommens, aber auch des Zusammentreffens oder Verfehlens spielt eine wichtige Rolle im Text: einmal als bekannter Weg, der den Reisenden sicheren Boden bietet und aus Sicht des Textes dem Schreibenden Gelegenheit gibt, sich als Kenner der Verhältnisse und Ortsansässigen und somit als zuverlässigen Erzähler zu erkennen zu geben, wenn er genaue Orts- und Wegangaben macht: "pero llegado a carigua que es doze leguas adelante de barquisimeto donde se toma el camino de cu- 
bagua pedro de linpias se alzo con la mayor parte de la gente y se fue camino de cubagua ${ }^{9}$ (R: 246). Der Weg kann aber auch als unheilvoller Wegbereiter des Unglücks fungieren, wenn Widersacher (Carvajal oder in einem Falle "yndios«, ebd.) ihr Opfer aufspüren. Manchmal muss der rechte Weg erst gefunden oder freigelegt werden (»cortar«, ebd.: 249). Er kann schließlich auch der Verständigung dienen, wenn verschiedene Teile der Truppe nicht zur selben Zeit am gleichen Ort sind: Zurückgelassene Briefe am Wegrand können die Kommunikation sicherstellen. Kommunikation kann zwei getrennte Gesprächspartner verbinden, andererseits aber auch manipulieren. Die Briefe am Wegrand dienten Carvajals Unterhändler Villegas dazu, Hutten in Carvajals Lager zu locken. Villegas sollte Hutten im Auftrag Carvajals suchen, fand ihn aber nicht und »dexole dos cartas en el camino con cavtela en [que, ms 3] solamente le hazia saver como bartolome belzer estaua en el tocuyo esperando el capitan general ${ }^{10}$ (R: 246). Die bewertende Konnotation des Wortes cavtela (>List $<$ ) macht deutlich: Die Geschichte wird nun aus Huttens Perspektive erzählt.

Um schnell nach El Tocuyo und zu Bartholomäus Welser zu gelangen, sei Hutten vorausgeeilt, als er die Briefe fand. In Barquisimeto sei er schließlich auf Villegas getroffen, der die Verhandlungen zwischen Hutten und seinem Widersacher Carvajal über einen weiteren Boten abgewickel habe. Der Erzähler betritt an dieser Stelle sozusagen den (Augen-)Zeugenstand: Die Sprecherinstanz wird wieder greifbar, eine Sprecherinstanz, die den Figuren ihrer Geschichte mit Einleitungssätzen mit verbum dicendi das Wort erteilt. Beides die wörtliche Ausgestaltung der Reden und die starke Präsenz der Sprecherinstanz - kann als Annäherung an eine notarielle und beglaubigende Erzählstrategie gedeutet werden, die den Eindruck einer wahrheitsgetreuen Aussage erzeugt und dem Schreiber erlaubt, sich selbst als glaubwürdig darzustellen.

Der verbale Schlagabtausch zwischen den beiden Rivalen bahnt sich bereits an, bevor diese persönlich zusammentreffen. Hutten, Welser und Ruiz de Vallejo befinden sich in Barquisimeto, während Carvajal etwas entfernt in seinem Lager weilt. Carvajals Positionen erfahren wir aus dem Munde des Juan de Villegas, einem seiner Gefolgsmänner, und des Klerikers Frutos de Tudela, den Carvajal als Boten eingesetzt hatte. Huttens Entgegnungen hingegen legt der Erzähler mehrheitlich diesem selbst in den Mund. Bereits die Reden des ersten Wortgefechts positionieren die beiden Gegner Carvajal und Hutten als Bösewicht beziehungsweise friedfertigen Helden: Carvajals Bote Villegas droht Hutten, Carvajal werde ihn mit 40 oder 50 Berittenen holen, 
wenn er nicht freiwillig $\mathrm{zu}$ ihm gehe: »[S]i no yba que el governador venia por el con 40/0 50 onbres da [wohl: de] a cauallo «" (R: 246). Hutten lehnt daraufhin explizit das Mittel der Waffengewalt zur Konfliktbeilegung ab: «[A] lo cual rrespondio felipe de huten esto no se a de librar a lanzadas yo no vengo a ver pasiones sino a hazer lo que su magestad mandara ${ }^{12}$ (R: 246). Da sie so zu keiner Einigung kommen, gibt Hutten vorerst nach und begibt sich in Carvajals Lager.

Im weiteren Verlauf wird Hutten als ein Edelmann konstruiert, der nicht einmal den Versuchungen erliegt, sich zu bereichern, mit denen ihm Carvajal - immer nach Vallejos Darstellung - aufwartet. Denn nachdem Hutten sein Vorhaben dargelegt hat, nach Coro zu ziehen, um dem König und dem Untersuchungsrichter über seine entrada Bericht zu erstatten, stellt ihm Carvajal die Beute von 4000 oder 5000 Pesos in Aussicht, die ihm die Entdeckung jenes Tals, von dem man Kenntnis habe, einbringen werde. Dem setzt Hutten entgegen, dass er niemals seinen Leuten das erbeutete Gold wegnähme. Obwohl nicht ausgesprochen, werden hier Carvajal niedere Beweggründe untergeschoben. Seine Loyalität gegenüber dem Kaiser betonend, wiederholt Hutten abermals, dass er diesem berichten und sich deshalb nach Coro begeben müsse. Carvajal erscheint so als derjenige, der die Pflichterfüllung gegenüber dem Kaiser unterbindet.

Am nächsten Tag kündigen Trommelwirbel (Carvajal »mando tocar vn atanbor«, R: 246) die Zuspitzung des Konflikts an. Die beiden gegnerischen Gruppen versammeln sich vor Carvajals Hütte. Die Figuren des Dramas so aufgestellt, legt der Erzähler Ruiz de Vallejo Philipp von Hutten folgende Rede in den Mund:

[S]eñor governador yo a cinco años poco menos que por mandado de su magestad sali de la cibdad de coro por capitan general e he ydo en descubrimiento de la probincia de tierra adentro con muchos travajos y perdidas asi mias como de todos los que fueron conmigo agora a querido dios que venga perdido no por mi culca (Sic) ni de los que conmigo fueron sino por ser desdichados ya v.m. vee como venimos de trocados [wohl: destrozados]/al servicio de su magestad conviene que yo y todos los que conmigo vinieron vamos a coro porque yo quiero yr a dar cuenta a su magestad de mi jornada y a su juez de rresidencia que me dizen que esta en coro por tanto a v.m. suplico que no me detenga y tanvien quiero dar cuenta de mi a los señores belzeres cuya es esta governacion por su magestad ${ }^{13}$ (R: 247) 
Diese Worte charakterisieren Philipp von Hutten als dem König treu ergebenes und seinen Männern verpflichtetes militärisches Oberhaupt; das bereits an früherer Stelle evozierte Bild des >Vaters aller Soldaten wird hier inhaltlich untermauert. Durch die wörtlichen Reden in diesem Teil des Briefes wird das Tempo der Erzählung drastisch gedrosselt, die Lesenden meinen, wie durch eine Lupe unmittelbaren Einblick in den Schauplatz des dramatischen Geschehens zu bekommen. Der Erzähler fährt fort: »[E]ntonces rrespondio carvajal sereis vosotros testigos como dize que esta governacion es de los belzeres aqui no tienen nada los belzeres sino su magestad ${ }^{14}$ (R: 247). Hier wird spürbar, dass sich der Konflikt zwischen den deutschen und den spanischen Eroberern an der Frage des Souveräns entzündet. Für die Spanier ist eindeutig König Karl V. das Oberhaupt, während sich die >Deutschen< zunächst als Untertanen der Welser und erst in zweiter Instanz des Königs bzw. Kaisers sehen. Als würde Hutten seine Unterlegenheit bereits ahnen, macht er seinem Gegner Zugeständnisse. Er räumt ein, dass er natürlich in erster Linie dem König Untertan ist: »[E]l capitan general rrespondio ya dixe primero que por su magestad $\ll^{15}$ (R: 247).

Das Wortgefecht driftet immer stärker $a b$ in Richtung der Frage der Legitimation der Macht; der Gebrauch der Wörter escribano (>Notar`) und asentar ('protokollieren`) soll demjenigen, der sie ausspricht, Macht und damit die Legitimation verleihen, Recht $\mathrm{zu}$ sprechen und Freiheitsstrafen $\mathrm{zu}$ verhängen. Interessant erscheint an dieser Stelle, dass Carvajal Hutten verhaften will, dies aber nicht direkt an Hutten gerichtet ausspricht, sondern indirekt über den Befehl an den Notar, die Verhaftung schriftlich festzuhalten: »[C]arvajal rrespondio calla no hableys mas ase[n]ta escribano que le mando que se vaya preso a su posada ${ }^{16}$ (R: 247). Überhaupt ersetzt Carvajal das Handeln durch Schreiben, wenn er Hutten nicht direkt festnimmt, sondern ihm indirekt über einen mündlich erteilten Schreibbefehl an den Notar gebietet, sich als Gefangener in die Hütte zu begeben. Hutten nimmt den Kampf mit Carvajal auf der von diesem betretenen Ebene auf, wenn er explizit äußert, ihn nicht als Richter anzuerkennen und dies auch offiziell protokolliert haben will: »[Y] el capitan general respondio asenta que apelo que no le conozco por juez que yo soy capitan general por su magestad y no e visto otra cosa contra ello ${ }^{17}$ (R: 247f.). Es kommt zu einem Handgemenge, das durch Huttens Auszug aus dem Lager unterbrochen wird.

Dass die Eskalation nur durch diesen Auszug zu verhindern ist, verheißt nichts Gutes: In den Savannen von Quibore macht der kleine Trupp um Hutten Halt, und weil man befürchtet, von Carvajal und seinen Leuten eingeholt 
zu werden, hält man sich in Stellung. Die Macht wird der Partei Carvajals zugeschrieben, wenn man befürchtet, von ihm verhaftet $\mathrm{zu}$ werden: »[P]orque nos temimos que carvajal venia a prendernos pusimonos a punto de esperar el rrequentro si nos lo quisiesen dar ${ }^{18}$ (R: 248). Unversehens gerät Hutten mit seinen Gefährten in die Fänge einer Gewalt, deren Legitimation zwar durchaus nicht geklärt ist, aber dessen ungeachtet sehr effektiv funktioniert.

Das befürchtete Einfallen Carvajals in ihr Lager trifft (vorerst) nicht ein. Stattdessen stößt eine Delegation Carvajals unter Juan de Villegas zu ihnen, um Frieden zu schließen. Das Ergebnis der Verhandlungen ist ein Friedensvertrag, der festhält, dass über das Vorgefallene weder ein Prozess abgehalten noch ein Bericht verfasst werden solle. Carvajal solle Hutten mit sieben Mann frei nach Coro ziehen lassen (vgl. ebd.: 248f.). Den Vertrag sollten alle Beteiligten, auch die Soldaten, unterschreiben. Die Schrift auf Papier ermöglichte, dass die Unterzeichnung des Vertrags nicht von Angesicht zu Angesicht vollzogen werden musste. Zunächst unterschrieben ihn Philipp von Hutten und seine Männer; Villegas nahm das unterzeichnete Dokument mit in das Lager von Carvajal, wo er und seine Gefährten ihre Unterschriften unter den Vertrag (die capitulos) setzten. Als letzten Satz der Vereinbarung zitiert Ruiz de Vallejo, dass sie "no yrian contra ellos [los capítulos] ni parte dellos so pena de traydores aleves y fementidos ${ }^{19}$ (R: 249). Dass Carvajal Hutten trotz des Vertrags überfiel, entlarvt ihn unmittelbar als »Verräter«. Wer den Vertrag bricht, soll als »Verräter« bestraft werden. In diesem Sinne nehmen die capítulos den Ausgang der Geschichte vorweg, die in Carvajals Verurteilung zum Tode endet.

Der letzte und auf die Enthauptung Huttens und Welsers hinauslaufende Teil des Konflikts wird mit dem Aufbrechen Huttens und seines Gefolges nach Coro eingeläutet (vgl. ebd.). Es stellt sich also ein Stück Weg zwischen das Ereignis der Unterzeichnung des Friedensvertrags und dessen Bruch. Die Bewältigung des Weges zögert das fatale Ende der Geschichte zwar etwas hinaus, weil sie das Zusammentreffen der beiden Gegner und damit den Überfall vorerst verhindert. Nach acht Tagesmärschen durch ebenes Land und zwei durch das Gebirge legt Hutten Rast ein; hier finden er und seine Leute Nahrungsmittel, sie können ihr Schuhwerk reparieren - die verbrauchten Schuhe als ein Zeichen für die Strapazen des Weges -, und die Pferde können sich ebenfalls stärken und für die Weiterreise rüsten. Am nächsten Tag bricht Hutten auf, nachdem er $18^{20}$ Leute vorausgeschickt hat, um den Weg freizumachen (vgl. ebd.). 
Die >Plötzlichkeit und der >Zufalk sind Merkmale, die das Erzählen des Überfalls prägen. Der Überfall ist ein Moment, der einen »Riß im normalen Ereignisverlauf" (Bachtin 2008: 15) markiert. Dieser »normale Ereignisverlauf "wird als betont friedlich und sorglos dem gewaltsamen Einfall vorausgeschickt und produziert so einen umso größeren Kontrast:

[E]l qual dia sobre tarde despues de rrancheados lloviendo media ora antes que el sol se pusiese llegó carvajal con mucha gente entre los quales vinieron casi todos los firmados quiso su ventura que con los primeros que toparon fueron con el general y bartolome belzer y muy descuidados estauan hechados en sus hamacas y ansi los prendieron sin darles lugar a levantarse como la cosa fue supita y se prendieron luego las dos cabezas no ovo hombre que pelease ni tomas armas yo quiso mi ventura que estaua algo desbiado del rrancho echando vnos clavos a mi cavallo ${ }^{21}$ (R: 249f.)

Bescheiden schreibt Ruiz de Vallejo dem Zufall (»ventura«) zu, dass er dem Überfall entkommen ist. Seine Handlungen sind Reaktionen darauf, was er von den Handlungen anderer hört und sieht (»oy«, »metime por ruido«, »veome« und unten »vide«):

[Y] como oy arma pense que eran yndios y tome vnas armas de plasencia, y un sayo suyo para rrodela y vna espada y metime por rruydo y veome cercar de cinco o seos hombres yo como vide la traycion determine de no me dar y en la verdad me mataran y [si, ms S. 7; SC] vn yndio mio no me socorriera con vna lanza pero todavia saque vna lanzada por la cabeza ${ }^{22}$ (R: 250).

Das Präsens (»veome«) hält dem Lesenden unmittelbar die Perspektive des Erzählers vor Augen: Die Bedrohung geht von den Verrätern aus, und Vallejos Entschluss, sich diesen nicht zu ergeben, sondern sie zu bekämpfen, zeichnet ihn als ehrenhaft und machtgetreuen Diener aus. Sein Überleben, an einem dünnen Faden hängend, verdankt er schließlich einem seiner Sklaven, dessen Treue zu Vallejo ebenfalls der Konstruktion als ehrenhaftem Mann zuträglich ist. Tapfer erträgt er auch noch die Wunde am Kopf, die ihm durch einen Lanzenstoß der Verräter beigebracht wurde. Die zahlreichen Verben der Wahrnehmung übermitteln Vallejo stets als Quelle der Informationen und rücken ihn als Augenzeugen und damit als vertrauenswürdigen Erzähler in den Vordergrund.

Den weiteren Verlauf der Ereignisse prägen Bewegungen auf verschiedenen bekannten und unbekannten Wegen der Provinz. Diese Ortsveränderun- 
gen teilen die Zeit, die Ruiz de Vallejo durchlebt, in verschiedene Abschnitte mit einzelnen Ereignissen ein.

[C] on todo llegue al rrancho del general por si le podria dar socorro vile preso y cercado a el y a bartolome belzer y yo entonces sali por vn monte y vine de noche a dar con los 18 hombres que adelante venia[n, ms S. 7] a cortary llegue a ellos a la media noche y alli nos parescio que seria lo mejor venir a coro a dar mandado al licenciado frias si aqui estuviese pues era governador y si no pasar por la via del cabo de la vela a santo domingo a dar mandado al señor cerrato deste caso $^{23}$ (R: 250).

Ruiz de Vallejo und seine Mitstreiter beginnen, die Zügel wieder in ihre Hand zu nehmen. Sie planen ihr Vorgehen. In Coro treffen die Männer plangemäß auf Frías, der die Verfolgung Carvajals sofort aufnehmen will. Wenige Tage später erreichen zwei »yndios« - ehemalige Sklaven Huttens - Coro. Sie konnten sich aus den Fängen Carvajals befreien und sich bis nach Coro durchschlagen. Ruiz de Vallejo lässt sie berichten, wie »aquella noche luego que prendieron al capitan general le cortaron la cabeza y a bartolome belzer y a gregorio de placencia y a un rromero ${ }^{24}$ (R: 250). Ruiz de Vallejo erfährt, dass Carvajal ihn suchen ließ: Die »Indianer« erzählen, dass Carvajal »por la mañana envio en mi busca y de los demás 50 hombres y a pedro de linpias por capitan y como no nos hallaron volvieronse ${ }^{25}$ (R: 250 ). Vallejo entkommt seinen Verfolgern ein letztes Mal mit Glück.

Was nun folgt, ist eine Bewertung der Tat Carvajals und eine Reflexion darüber, was diese Hinrichtung und der Untergang Philipps von Hutten für die Provinz bedeutet. Die Edition von 1961 transkribiert allerdings fehlerhaft: "[N]o sabemos que pensar de carvajal aver hecho lo que a hecho sobre traycion pensaba« - im Manuskript des AGI heißt es aber: »sobre traycion pensada« (AGI, Justicia, Leg. 992, $\mathrm{N}^{\circ} 4,3^{\mathrm{a}}, 17 \mathrm{v}^{\circ}-20 \mathrm{v}^{\circ}$, S. 7; Hervorhebung SG) ${ }^{26}$ (R: 250). An dieser Stelle kommen - sonst nirgends so ausführlich dargelegt - angebliche Pläne zur Sprache, die Hutten für die Provinz gehegt haben soll. Er habe zehn oder zwölf Dukaten ausgeben wollen, um Leute, Kleider, Vieh und Pferde aus Kastilien zu holen; er habe drei Dörfer gründen und Beroberoata besiedeln wollen. Danach habe er vorgehabt, sich über die Nachricht der Omagua, des kriegerischen Volks am Fluss Marañón (i.e. Orinoco, vgl. Schmitt/Simmer 1999: 108), Gewissheit zu verschaffen (vgl. R: 250). Insgesamt glaubt Ruiz de Vallejo, dass Philipp seine Pläne verwirklicht hätte, wenn er am Leben geblieben wäre, denn er habe mehr Geld ausgeben wollen als alle, die mit ihm gekommen seien. Aus diesem Grund hätte der König Hutten die Provinz auch 
nicht entzogen. Zuletzt wird die Schuldfrage angesprochen: »[T]oda la culpa desto no la echan en esta tierra a nadie sino es al señor licenciado cerrato presidente (ebd.: 251). Gemeint ist damit der Präsident der audiencia ${ }^{27}$ von Santo Domingo; inwiefern ihn die Schuld treffen soll, wird nicht erläutert. Es ist eine Fährte, die auch im Kriminalprozess gegen Carvajal nicht wieder aufgenommen wird.

Der Sprachgebrauch rückt den Brief in die Nähe der Rechtspraxis. Damit bildet er eine Art Scharnier zwischen Huttens Briefen und den Prozessakten. Der Archivierungsort im AGI - der Brief wird in den Akten des Amtsprüfungsverfahrens gegen Carvajal aufbewahrt - weist ihm diesen Kontext ebenfalls zu. Als dem Bereich des Rechts zugehörig weisen den Brief nicht nur seine archivarischen, sondern auch seine inhaltlichen und textstrukturellen Merkmale aus: Rückblickend legt Ruiz de Vallejo die blutige Auseinandersetzung zwischen Hutten und Carvajal während der Hutten-entrada aus seiner Sicht als Augenzeuge dar. Er weist klar die Schuld zu beziehungsweise entlässt sich aus der Verantwortung, und der Text könnte gut und gern als Mitschrift einer Zeugenaussage des Prozesses durchgehen.

Bezogen auf die koloniale (Schreib-)Situation zeigt der Brief des Landsknechts Ruiz de Vallejo ihn als Schreiber, der sich und seine Anliegen zu präsentieren weiß. Das sich unterwerfende koloniale Subjekt wurde anhand Carvajals Vorverurteilung durch Juan Pérez de Tolosa angerufen und spricht im Brief vollständig im Sinne des Richters. Dadurch, dass er dem Opfer des >Bösewichts ein Denkmal konstruiert, verschafft sich Ruiz de Vallejo Gelegenheit, sich selbst im gleichen Atemzug in gutem Licht erscheinen zu lassen und sein Handeln als den Umständen angemessen zu rechtfertigen. Dass die indigenen Gruppen nur am Rande erwähnt werden, weist den Text ebenfalls als >koloniak aus. Ihre textuelle Marginalisierung wird zum stummen, impliziten Bedeutungsträger, der das Liefern von expliziten Argumenten für die Überlegenheit der Europäer überflüssig macht.

\section{Galeotto Cei: Viaggio e relazione delle Indie}

Dieser Abschnitt konzentriert sich auf die Beschreibung und Analyse derjenigen Passagen in Galeotto Ceis 161 Seiten starkem Bericht, die sich um den Konflikt zwischen Hutten und Carvajal drehen. Das Manuskript wird im British Museum in London aufbewahrt (vgl. Surdich 1992: IV) und wurde erst 1992 unter dem Titel Viaggio e relazione delle Indie (1539-1552) von Francesco Sur- 
dich herausgegeben (vgl. $C^{28}$ ). Diese Edition ist auch Grundlage der hier präsentierten Analyse.

Gemäß den einleitenden Worten des Herausgebers hielt sich der aus einem florentinischen Handelsgeschlecht stammende Galeotto Cei (1513-1579) in den Jahren zwischen 1539 und 1552 in Venezuela auf. Sein Vater hatte sich in den ersten Jahren des 16. Jahrhunderts vom Regime der Medici distanziert, woraufhin er verhaftet, gefoltert und schließlich hingerichtet wurde (vgl. Surdich 1992: I). In Lione, wo sich Galeotto und dessen Onkel seit 1529 aufhielten, wurde der Familienbesitz durch die Medici konfisziert. Nach der Schlacht von Montemurlo geriet Galeotto Cei in Pisa in Gefangenschaft und wurde nach der Intervention der Herzogin von Parma und einer »einflussreichen spanischen Persönlichkeit, Lop [sic!] Hurtado de Mendoza« (ebd.) 1538 freigelassen und nach Spanien verbannt. Dort schloss er einen Handelsvertrag mit Luigi da Ricasoli ab.

Mit diesem Vertrag in der Tasche reiste er nach le Indie wo er - ähnlich wie Hutten, dessen Vertrag mit den Welsern ebenfalls auf einer ökonomischen Grundlage beruhte - sein Glück suchte in der Hoffnung, zu Reichtum zu kommen. Wie Hutten blieb jedoch auch ihm der Geldsegen verwehrt er kehrte nach 14 Jahren ohne großen Schatz (»senza un gran thesoro«, C: 1) nach Hause zurück. Über diese Reise verfasste er nach seiner Rückkehr nach Florenz einen 161-seitigen, unvollendeten Bericht, den er an einen florentinischen Händler richtete: „Al molto magnifico et suo honorabile messer Bartolomeo Delbene« (ebd.: 1). Delbene war seinerseits verwandt mit dem bekannteren Niccolò del Benino, der in Potosí an der Ausbeutung von Minen beteiligt war. Jener Niccolò del Benino und Galeotto Cei müssen in las Indias- so Amanda Salvioni (vgl. 2004: 167f.) - Freundschaft geschlossen und auch nach der Rückkehr nach Europa in Verbindung gestanden haben, wie ein Brief, den Niccolò an Cei gerichtet habe, beweise. Der vertrauliche Ton in jenem Brief, aber auch in Ceis Viaggio e relazione delle Indie zeige die engen Verbindungen auf, die zwischen den Mitgliedern der florentinischen Handelsschicht und den Interessen in le Indie bestanden hätten.

Was heißt das nun für die Interpellation, für die Anbindung an den kolonialen Apparat und damit die Prägung des Schreibens von Galeotto Cei? Nach Salvioni müsste sich dieses grundlegend anders gestalten als in Texten, die an einen offiziellen, institutionellen Adressaten gerichtet waren:

La existencia de dichos vínculos [entre miembros de la clase mercantil florentina con intereses en las Indias], aludida continuamente [en ambos tex- 
tos], explica el hecho de que Cei dedicara su obra a un par suyo, y no a un destinatario institucional, ligado a la política colonial, como ocurre en la mayoría de los testimonios de viajeros españoles. ${ }^{29}$ (Ebd.: 168)

Wenn die Hierarchie zwischen Schreiber und Adressat keine Rolle spielt, dann heißt das keineswegs, dass der Modus des kolonialen Diskurses ausgeschaltet ist, denn beide gehören der Klasse der Kolonisierenden an, auch wenn sie nicht direkt dem spanischen Hof angeschlossen sind. Außerdem kennen wir zwar den Adressaten, nicht jedoch den genaueren Hintergrund und Anlass des Schreibens. Bereits ein erster Blick auf den Bericht des Florentiners zeigt: Wie die anderen Texte, die aus der kolonialen Situation hervorgehen, produziert auch Ceis Relazione ein koloniales Subjekt, das auf Unterwerfung und Ausbeutung der >entdeckten 〈 Völker aus ist. Sein Operieren mit der »Differenz zwischen Beherrschern und Beherrschten « (Kiening 2003: 187), nach Kiening eines der Merkmale des kolonialen Diskurses, ist allgegenwärtig.

Die Debatte der Kolonialforschung gänzlich außer Acht lassend, behauptet Rinaldi in einem Band über florentinische Seefahrer mit dem Titel Oceano Arno - i navigatori fiorentini, dass diese geprägt gewesen seien von einem $\mathrm{Hu}$ manismus, der sie zu Vorkämpfern gegen den Rassismus gemacht habe (vgl. Rinaldi 2012: 27). Wie dieser angebliche Humanismus allerdings zu seiner Beobachtung passt, Cei habe den Sklavenhandel als Gelegenheit gesehen, Profit zu schlagen, ohne ihn zu verurteilen (vgl. ebd.: 153), was wiederum auf eine Beteiligung Ceis schließen lasse, lässt er unbeantwortet.

Sogar in der Einleitung zum Band (vgl. Marrani 2012), die grundlegend in die Thematik der florentinischen Seefahrt einführen soll, fehlen die Bezüge zur Kolonialforschung. Im Gegenteil: Mit nicht wenig Stolz führt Marrani aus, die florentinischen Handelshäuser und Banquiers seien in gewisser Weise die Unterstützer der überseeischen Abenteuer des großen Zeitalters der Entdeckungen (»avventure oceaniche dell'età delle grandi scoperte geografiche«, ebd.) gewesen. Auch sei bisher wenig bekannt, dass Florenz neben Lissabon und Sevilla eine zentrale Rolle bei den Entdeckungsfahrten des 15. und 16. Jahrhunderts gespielt habe (vgl. ebd.: 11). Insbesondere der Bereich der Kosmographie habe Figuren vom Format des bekannten Paolo Dal Pozzo Toscanelli hervorgebracht. Auf dessen Schule - Toscanelli hatte $1474 \mathrm{im}$ Auftrag des portugiesischen Königs Alfons V. eine Karte erstellt - hätten sich sowohl Christoph Kolumbus als auch Amerigo Vespucci bezogen (vgl. ebd.). Rinaldi sieht die florentinischen Seefahrer gerne in einer Reihe mit den spanischen und portugiesischen >Entdeckern . Obwohl Florenz nicht am Meer 
liege, hätten es seine Bewohner verstanden, nautische Karten und »mappamondi« (Rinaldi 2012: 19) zu lesen. An den Ufern des Arno habe sich eine wahrhafte "scuola di navigatori« (ebd.) herausgebildet, die Wege in die weite Welt eröffnet habe. Kennzeichnend sei für diese Seefahrer gewesen, dass sie ihre Reisen als spirituelle Übung verstanden hätten, zu der auch die Berichterstattung gehört habe: »[I]l viaggio era atto vano, misero e raggrinzito se non si faceva racconto, relazione, trasmissione, storia «o (ebd.: 21). Allesamt seien die Seefahrer aus Florenz gebildete Männer gewesen. Dass sie mehr geschrieben haben sollen als andere, wie der Autor behauptet, weil Florenz ihnen »una lingua, svelta nella parlata, raffinata nella scrittura« (ebd.: 23) geschenkt habe, lässt sich kaum aufrechterhalten; die prall gefüllten Archive in Spanien (und Portugal) sprechen eine andere Sprache. Wie dieses Kapitel zeigen wird, steht Ceis Text den anderen in Bezug auf die Motive der Reise in nichts nach, was ihn als dem kolonialen Diskurs zugehörig ausweist.

Galeotto Cei wurde auf unfreiwillige Weise Zeuge von den Geschehnissen in der Provinz Venezuela. Genau wie Bartholomäus Welser und Philipp von Hutten wurde er im Jahr 1545 von Juan de Carvajal an der Weiterreise gehindert und in der Folge in der Provinz festgehalten. Erst 1551 erhielt er von Juan Pérez de Tolosas Nachfolger die Erlaubnis, auszureisen (vgl. Schmitt/Simmer 1999: 69, 154f.). Cei war nach Coro gekommen, weil er - wiederum wie Hutten - Opfer von Gerüchten geworden war, die Glauben machten, dass die Provinz über Gold verfüge. Cei erreichte die Neuigkeit in Santo Domingo, dass »era arrivata certa gente con un capitano che tornava da discoprire et che portavano 20 mila ducati in oro, che in tale provincia era gran cosa, et venivano tutti nudi et disarmati, con voluntà di tornare di nuovo a discoprire et popolare « ${ }^{31}$ (C: 40). Wie ein böses Omen gesellt sich in den Berichten über spektakuläre Goldfunde das Prekäre, das Heruntergekommene, die Armut, sichtbar darin, dass die Goldsucher alle "nackt und unbewaffnet " gekommen seien.

Ceis Reisebericht gleicht in vielen Punkten den Briefen Philipps von Hutten. Von Hunger, Durst und sintflutartigen Regenfällen ist zu lesen; auch Cei berichtet im selben Atemzug von zelebrierten Messen und Raubzügen gegen die »Indi ${ }^{32}$ «, denen Cei und seine Gefährten Mais, aber auch Baumwollfaden abnahmen, um Schuhe und Pferdegeschirr herzustellen: »I giorni di festa un prete ci diceva messa et passavamo tempo a cavallo; andavamo a discoprire et rubare que poveri Indi 10 e 15 leghe intorno: el maggior rubo era filo di canapa et cotone per fare scarpe et fune per li cavalli..133 (C: 44) Die berüchtigte und in Huttens Briefen stetig wiederkehrende Jagd nach »Indiern« prägt auch Ceis 
Schreiben: »[E] andammo 20 compagni a discoprire e cercare di pigliare Indi per servizio ${ }^{34}$ (ebd.).

Galeotto Cei berichtet von der Umsiedlung eines großen Teils der Bewohner von Coro nach El Tocuyo, zu der Carvajal die Leute gezwungen hatte. Er erzählt, dass sie Hütten aus Schilfrohr (»canne, paglia et legnami«, ebd.: 45) bauten und dass sie in der Folge mit Hunger zu kämpfen hatten. Diesen hätten sie mit Raubzügen nach Mais bekämpft, die Cei beschönigend als >Funde bezeichnet: Seine Mitreisenden und er hätten zwar große Mengen an Mais in der Gegend ‘gefunden<, der sei aber noch grün gewesen; zudem hätten sie es vernachlässigt, den Mais vor den Papageien zu schützen, die ihnen die Hälfte weggefressen hätten. So hätten sie alle 15 Tage auf Raubzug gehen müssen (»andare a rubare«, ebd.). Sie seien am Anfang halb barfuß gegangen, die Füße in Hirschleder eingewickelt, und hätten sich von nichts anderem als Brot und Wasser ernährt (vgl. ebd.). Hunger dient als Rechtfertigung der Raubzüge gegen die »Indi«, bei denen sich die »Cristiani« offenbar nach Bedarf bedienten.

Hutten schreibt seine Briefe, während er unmittelbar im Geschehen steckt. Im Gegensatz dazu schreibt Cei seinen Bericht aus zeitlicher Distanz; Amanda Salvioni (vgl. 2004: 167) datiert ihn aufgrund textinterner Hinweise auf das Jahr 1556. Da Cei also zum Zeitpunkt der Niederschrift am Ende der Ereignisse steht, die er aufschreiben will, kann er sie jeweils einzeln daraufhin bewerten und entsprechend gewichten. Huttens eher holpriger Schreibstil hebt sich vom fast >photographisch der - so Rinaldi (vgl. 2012: 151f.) - den europäischen Leserinnen und Lesern unbekannte Dinge illustrieren wollte.

Während Rinaldi Ceis Vorreiterrolle für die Ethnographie in positivem Sinne hervorhebt, gehört für Gabriel R. Ricci Ethnographie und »literary colonialism « zusammen: »In Homer's Odyssey we have inherited an original template for the travel of discovery, personal transformation and the moral triumph of the individual in the face of cosmic irony, and unremitting misadventure and obstinate adversity. (Ricci 2014a: viii) Nach Homers Odyssee und Herodots Historien folge

a torrent of travel writing that has accompanied exploration and imperial conquest, chronicled the Grand Tour, kept pace with pious pilgrimages and missionary zealots, registered exotic botanical appropriations and enlivened natural history, produces a literary colonialism, and enhanced the field of ethnography. (Ebd.: viiif.) 
Sowohl Ceis als auch Huttens Schreiben passen auf diese Schablone: Die Inbesitznahme des Raumes durch das Nachzeichnen der Fortbewegung, die Einsprengsel der Naturbeschreibungen, aber auch die ständigen Widrigkeiten, die überwunden werden müssen, zeichnen beide Texte aus. Wie andere Berichterstatter erwähnt er die zahlreichen Sprachen und Nationen des durchquerten Landes. Ihr Wert wird bemessen an der Menge an Gold, das ihnen abgenommen werden konnte: "[I]n 7 anni, in 40 leghe di circuito, no se ne cavò mille ducati doro, con tutte l'estorsione et rubi vi si feciono « ${ }^{35}$ (C:45). Und wie zahlreiche andere berichtet auch er über kannibalistische Bräuche der Einheimischen, ohne eine Quelle seines >Wissens` anzugeben.

In der Folge werden das Land beschrieben, das Klima, die plötzlichen und ruinösen Regenfälle, die die Flüsse in Kürze anschwellen ließen, wie die »Indi« des Landesinneren Salz oder auch Hanffaden herstellten, den sie an andere »Indi« und »Cristiani« verkauften. »Questi Indi che fanno il sale non seminano, ma solo attendoneo a esso et cavare di quella coccuiza et farne filo di quelle sete o amacche in che dormono, e lo vendono alli Indi e a'Cristiani per maiz et sempre ne hanno gran quantità « ${ }^{36}$ (ebd.: 46). Solche und ähnliche Beschreibungen der Geographie, des Klimas und der Bräuche der autochthonen Bevölkerung sind hier wie dort Bestandteile einer keineswegs unschuldigen Textgattung: der »Reiseliteratur«, die Gabriel R. Ricci im Gegensatz zu Rinaldi durchaus von Interessen geleitet sieht. So schreibt Ricci in seiner Einleitung zum Band Travel, Discovery, Transformation: "Travel and the history of travel writing take in multiple pursuits: exploration and conquest, religious pilgrimage and missionary work, educational tourism and diplomacy, scientific and personal discovery, and natural history, and global botanizing, to name just a few« (Ricci 2014a: vii).

Sich wieder den Geschehnissen zuwendend, verlässt Cei den beschreibend-ethnographischen Modus, der dann fast pikareske Züge annimmt: Der aus edler Familie stammende Galeotto Cei beginnt, die Milch seiner Kühe zu Käse zu verarbeiten und Hühner zu halten. Er muss nur darauf warten, dass letztere Eier legen (vgl. C: 46).

Die Erzählung nähert sich langsam, aber sicher dem Moment der Begegnung Ceis mit Philipp von Hutten und Bartholomäus Welser. Die blutigen Auseinandersetzungen zwischen den Fraktionen der Eroberer ${ }^{37}$ kündigen sich an, indem Carvajal sein Versprechen, Cei nach der Umsiedlung weiterziehen zu lassen, nicht einhält, sondern seine Einlösung auf nächstes Jahr verschiebt. Er wolle zuerst erkunden, ob es in dieser Gegend »alcuna ricchezza« (ebd.) gäbe, und danach die Erlaubnis erteilen. Er bereitete den Erkundungs- 
zug mit 80 Leuten vor und plante, $40 \mathrm{im}$ Dorf zu lassen. Unterwegs vernahmen sie von den »Indi«, dass in 20 Ligen Entfernung »Cristiani« vorbeizogen (vgl. ebd.). Diese »Cristiani« erreichten das Dorf Ende Februar 1546: Es waren Bartholomäus Welser mit 20 Gefährten, »tutti malvestiti, con//solamente dua cavalli« (ebd.). Sie seien von ihrem Generalkapitän Philipp von Hutten in die Gegend von Coro oder nach Santo Domingo vorausgeschickt worden, um Leute von dort $\mathrm{zu}$ holen. Die von Cei nachgezeichnete Geschichte stimmt in den Grundzügen mit den anderen Texten des Korpus überein. Auch Cei bezichtigt Carvajal purer Bosheit, als dieser »per malvagità« (ebd.) Welser die Erlaubnis verweigert habe, zu seinem Kapitän Philipp von Hutten zurückzukehren oder den Weg zum Hafen nach Coro fortzusetzen, wohlwissend, dass »in Santo Domingo, aute [wohl: ante; SG] queste nuove, non potevono lasciare di levarli el carico, tornarlo a dare a costui, come provisto dal consiglio di Spagna ${ }^{38}$ (C: 47). Carvajal habe sofort einen seiner Stellvertreter mit 60 Mann auf die Suche nach Hutten geschickt, den sie dann am Karsamstag $1546 \mathrm{zu}$ ihm ins Lager gebracht hätten.

An dieser Stelle lässt sich eine weitere Form des kolonialen Schreibens ausmachen, indem sich Cei als koloniales Subjekt präsentiert, indem sich das Ich auf die Seite der Gegner Carvajals schlägt:

[S]imilmente ne parlai al nostro governatore dimostrandoli come era malvolsuto dalla sua gente e che in ritenere quivi costoro dava loro un capo et che il meglio suo saria lasciarli andare al loro viaggio et accordarsi con loro di lasciare loro la terra e, in fin d'ottobre, con assai bestiami che lui haveva mezzi rubati e altri poteva comperare con argenti lavorati haveva, se ne andasse con sua amici al Nuovo Regno di Granata, dove haria fatto 15 o 20 mila ducati. Non mi volse credere, ma fecie fare un ceppo per metterli in prigione et così assicurarsi di loro ${ }^{39}$ (C: 47).

»Er wollte mir nicht glauben « - diese Aussage soll darauf verweisen, dass Cei die Lage damals richtig, das heißt im Sinne der späteren Verurteilung, eingeschätzt und dies auch bekundet hatte. Doch Hutten und seine Leute wollten Cei, gemäß dessen Darstellung, nicht glauben, als er sie gewarnt hatte, dass Carvajal und seine Leute den ausgehandelten Waffenstillstand nicht einhalten würden (vgl. ebd.: 48). Immer wieder wird der Satz: »Non mi volsono credere«, wiederholt, womit Cei sich selbst als denjenigen darstellt, der alles vorausgesagt haben will: »[E]t Iddio mi sia testimone se non lo predissi tutto quello li accadde $\ll^{40}$ (C: 48$)$. 
Die Hinrichtungsszene folgt gleich darauf; erstaunlicherweise wurde diese Textstelle in der Edition Schmitt/Simmer nicht aufgenommen. Gemäß Cei lag der Ort des Geschehens 40 Meilen von ihrem Dorf (wohl: El Tocuyo) entfernt in einer hinter großen Steinen verborgenen Schlucht. Dort habe Carvajal ohne Hindernis zwei »Cristiani« ergriffen und ihnen sofort den Kopf abgetrennt. ${ }^{41}$

Interessant ist, dass sich in den Schilderungen der Hinrichtungsszene in den einzelnen Texten jeweils die gleichen Elemente teilweise wortwörtlich wiederholen: dass Carvajal die friedlich lagernden Leute um Philipp von Hutten mit Leichtigkeit überwältigte; den Männern »hemmungslos« (»incontinente «) den Kopf abtrennte; dass er ihnen entgegen dem katholischen Brauchtum der Spanier die Beichte verweigerte, obwohl ein Priester zugegen war:

[E]t di poi, incontinente, fece il simile a Bartolomeo Belzeri et a Filipo de Hutten, non avendo rispetto che fusse assai imparentado con la casa d'Austria, né solo concesse loro si confessassino, sendo un preto con loro, cosa molto contro al costume spagniulo, che sono molto catholici in apparenzia, ma la rabbia li fece dimenticare la prospettiva loro ${ }^{42}$ (ebd.: 48 ).

Diese Übereinstimmungen könnten ein Hinweis darauf sein, dass der Vorfall Gesprächsthema war und sich so mit der Zeit als Diskurs verfestigte. Ceis Hinweis auf die Ungeheuerlichkeit, einen Mann umzubringen, der mit dem Königshaus der Habsburger verschwägert war, fehlt allerdings in allen anderen Texten. Woher Cei seine Informationen hatte - ob er der Szene als Augenzeuge beiwohnte oder sie aus Erzählungen und Berichten anderer, zum Beispiel der »India Magdalena« oder »Perico«, kannte -, sagt er nicht. Aufgrund der fehlenden Details ist jedoch zu vermuten, dass er von der Enthauptung der vier Männer nur indirekt erfahren hatte. Man kann mit Salvioni einiggehen, dass in der Darstellung der Enthauptungsszene Las Casas' vernichtende Kritik an der spanischen Conquista durchschimmert, festgemacht am merkantilen Unternehmen, in dem Cei involviert war: "También en este caso, la codicia y salvajismo de los españoles, en la vision de Cei, es obstáculo para la explotación y la rentable administración del territorio «33 (Salvioni 2004: 177). Ceis Kritik an der spanischen Konquista ist also nicht als die einer >humanistischen Gesinnung Entsprungene misszuverstehen.

Ceis Bericht eröffnet nun die Perspektive derjenigen, die nach den Hinrichtungen im Lager Carvajals ausharrten. Er malt das Bild einer eigentlichen Terrorherrschaft: Carvajal ließ gemäß Cei einige der Männer in Ketten legen 
und habe hin- und herüberlegt, ob er ein weiteres Dutzend von ihnen enthaupten oder erhängen sollte (vgl. C: 48). Ihm selbst habe Carvajal nach dem Leben getrachtet, indem dieser versucht habe, ihn nachts im Bett umzubringen. Er sei aber gewarnt worden und habe deshalb wiederum ganz nach der Art eines pícaro nicht mehr zu Hause, sondern einmal im Röhricht, einmal im Wald, einmal bei dem einen, einmal bei dem anderen Freund übernachtet. Dieses Leben habe vier Monate gedauert. Carvajal habe geplant, den Gefangenen und den Toten den Prozess zu machen; Letztere habe er des Diebstahls und des Verrats bezichtigt. Weiter habe er beschlossen, acht Personen hinzurichten, denen er ihr Hab und Gut entzogen habe (vgl. ebd.: 49).

Das Eintreffen des "guidice di residenzia« (ebd.) - Juez de Residencia »Juan Perez di Tolosa « wird als markantes Ereignis hervorgehoben, das Carvajals üblen Machenschaften ein Ende setzte. Pérez de Tolosa sei im Morgengrauen des 27. August mit 60 Männern der ursprünglichen Truppe von Philipp von Hutten angekommen. Er habe in Coro von den Hinrichtungen erfahren und sich sofort auf den Weg gemacht, um Carvajal gefangen zu nehmen und dessen Flucht zu verhindern. Wieder habe Carvajal einer Nachricht - dieses Mal übermittelt von den »Indi« - nicht geglaubt, was ihm zum Verhängnis wurde, denn so konnte er ohne größeren Widerstand mit seinen Anhängern verhaftet werden: »Ancora l'Indi havessini detto vengono Cristiani, el nostro governatore non lo voleva credere et se li trovò addosso senza pensarvi et subito fu preso con li sua seguaci« (ebd.).

Die Modellierung der Figur Carvajals als Despot gipfelt im Vorwurf der Urkundenfälschung: Man habe nach seiner Verhaftung eine königliche Urkunde bei ihm gefunden, die Carvajal selber gefälscht und in der er sich selbst Autorität verschafft habe, die ihm in Wirklichkeit nicht zuzurechnen sei (»piena autorità, che non haveva in effetto«, ebd.). Auch habe er eine Liste mit acht Personen mit sich geführt, die er habe umbringen wollen, auf der er, Cei, an zweiter Stelle gestanden habe. Auf Ceis Frage, weshalb Carvajal ihn habe umbringen wollen, habe er geantwortet, weil Cei ihm die Wahrheit gesagt habe und wegen einer gewissen Frauengeschichte (vgl. ebd.). Durch diese nichtigen Begründungen für ein Todesurteil, die Cei Carvajal in den Mund legt, lässt er ihn in noch ungünstigerem Licht erscheinen, während Cei sich demgegenüber als wahrheitsliebender und aufrichtiger Untertan beweisen kann. Mit der Wiedergabe des Prozesses bestätigt er dessen Rechtmäßigkeit und die gerechte Strafe durch den Strick, zu der Carvajal verurteilt wurde. Die Ehre der Toten sei wiederhergestellt worden, ihre Güter - vier Lumpen und zwei Pferde - seien ihnen zurückerstattet worden; Carvajals Gefährten seien aus- 
gewiesen worden (vgl. ebd.). Obwohl kein direkter Untertan der spanischen Krone, schreibt sich Cei als koloniales Subjekt in den bürokratischen Apparat ein - die Interpellation scheint auch hier zu greifen.

Galeotto Cei hat mit Philipp von Hutten gemein, dass er in der Ferne das Glück - Reichtum - suchte und dass am Ende der Reise der Misserfolg steht. Cei schreibt in den ersten Zeilen seines Berichts, dass er "senza un gran thesoro«, aber doch immerhin heil zurückgekehrt sei (zitiert nach Rinaldi 2012: 138). Er zeigt sich darüber pragmatisch-glücklich, denn von 100 Mann kehrten nur deren zwei zurück, die sich darüber glücklich wähnen dürften (vgl. ebd.: 139). Die »Cristiani« in »'India« seien allesamt sehr arm, und fast möchte man meinen, Cei habe Philipp von Hutten vor Augen gehabt bei der Bemerkung, sie hätten es vorgezogen, sich entbehrungsreich durchzuschlagen, als arm nach Hause zurückzukehren (vgl. ebd.: 138).

Beide Texte transportieren den »código mercantil«, den Beatriz Pastor in den Texten der Reiseliteratur ausmacht, dem Genre, das bereits im 16. Jahrhundert mit der europäischen Expansion kodifiziert und ausgebildet gewesen sei (zitiert nach Salvioni 2004: 181). Es handle sich um informative Texte, geprägt von einer spezifischen Rhetorik der Beschreibung, kombiniert mit einer gewissen literarischen Intention. Die Reiseberichte insbesondere der spanischen und italienischen Reisenden seien geprägt gewesen von einem spezifischen Code (»forma textual codificada«, ebd.: 182), der in Spanien durch Anordnungen und rhetorische Vorschriften des Imperiums (»leyes retóricas impuestas por el imperio«, ebd.) nach und nach formalisiert worden sei, während die italienischen Texte die alte Tradition evident(er) bewahrt hätten (vgl. ebd.: 181). So sei Ceis Text zwar nach utilitaristischen Kriterien angeordnet, die der merkantilen Logik entsprungen seien, zeige aber auch Spuren vorangehender Lektüren wie beispielsweise Vespuccis Mundus Novus, Las Casas' Vulgata oder Dante, dessen Einfluss die Autorin in der Beschreibung der Schlucht, in der die Enthauptung stattfindet, sieht: »un burrone fra grandissime pietre« (C: 48) liest sie als ein »infierno dantesco« (Salvioni 2004: 176). Insgesamt zeige der italienische Text eine größere Unabhängigkeit gegenüber den textuellen Vorgaben, denen sich die spanischen Schreibenden in stärkerem Maße zu unterwerfen hatten: Während der Spanier im Modus der "probanza de méritos y servicios « ${ }^{44}$ (ebd.) zu schreiben und sich so an einen politisch-institutionellen Leser zu wenden hatte, richtete sich der Italiener an ein neugieriges und heiteres Publikum mit dem Ziel, dieses zu unterhalten (»per tenere di che ridere«, zitiert nach ebd.: 177). Solche Komik »casi boccacciana« (ebd.: 177) sieht Salvioni in der populären florentinischen Literaturtra- 
dition verankert. Ein Beispiel hiefür ist folgende Episode, von Cei als »cosa da ridere« bezeichnet:

[S]tando un giorno in aguato solo, a un laghetto dove solevano venire Indi per acqua, per vedere se potevo pigliare Indio o India perché c'insegnassi dove stavono li altri nascosti et ci servissi di guida, et li altri Cristiani erono ripartiti in altri luoghi circumvicini, per simile effetto accadde che, dove io ero, venne una India per acqua et per discoprire ancora se vi eravamo, et era di più di 30 anni: quando fu sul lagho, mi scoprii et la presi et lei si cominciò a difendere e fare forza per non venire meco et, non volendo ammazarla, nè farli male, andavo agevolandola, infine, quando vedde non potere uscirmi dalle mani, si pose una mano al sedere et, scaricando el ventre, m'impaniò con essa tutto el viso, di modo che, non che lasciarla, per il puzo mi gettai nel laghetto a capo innanzi, dove al romoro corsono li altri et feci loro di me uno bello spettacolo, e la India si fuggì et vi fu che ridere; et questo adviene alcuna volta perché è tenuta vile cosa ferire o ammazzare femmine (C: 44 ).

In dieser Episode bekommt nicht nur eine ganz bestimmte »India«, sondern auch der Protagonist ein Gesicht. Dieser macht sich in einzigartiger Weise zum Ziel des Gespötts sowohl seiner Gefährten als auch der »India«, die ihn überlistet und ihn auch noch verlacht. Trotzdem zeigt sich der Text in der Szene eindeutig als kolonialer Diskurs, indem er eine klare Differenz zwischen dem Protagonisten Cei und der »India«, zwischen dem kolonialisierenden Subjekt und dem kolonialisierten Anderen markiert: Der ekelerregende Trick, den die »femmina« anwendet, markiert sie mit einer Differenz zu den Europäern und weist sie als Wilde aus. Während in den anderen Texten unseres Korpus das Jagen und Töten von indigenen Gruppen als nicht weiter kommentierte Selbstverständlichkeit daherkommt, gesellt sich hier eine groteske Note dazu, was dem Text einen eigenartig makabren Anstrich verleiht.

Den Topos der »beffa« (〉Spottく, >Ironie`) - Huttens Briefen vollkommen fremd - arbeitet auch Hester (vgl. 2014: 246) als Merkmal der Relazione heraus, weist es allerdings dem Einfluss des Decamerone zu. "Cei uses the beffa trope not not for the purposes of light entertainment but to portray a world too brutal and violent for the gap between Self and Other, between European and non-European, to be bridged « (ebd.). Dazu gehört auch das bereits beschriebene enge Nebeneinander von Komik, Schrecken und Leiden.

Auch Hester sieht hinter Ceis Schreiben toskanische Modelle, insbesondere in den persönlicheren oder anekdotischen Momenten der Erzählung (vgl. ebd.: 245). Denkt man an die von Cei erzählte Episode mit der »India« zurück, die ihn dem allgemeinen Gelächter aussetzt, kann man sich fragen, weshalb 
Cei sie überhaupt aufschreibt. Hester sieht die Antwort in einer Verbindung zur literarischen Textwelt: zu den Frauen, die im Decamerone die Männer verspotten. In diesem Sinne liefert dieser Text ein diskursives Modell für Ceis Erzählung. »Coming from a culture that celebrates Boccaccian novelle replete with women who play jokes on men and with scatological elements, he [Cei; SG] can only interpret the incident as funny, literary and, therefore, worthy of recounting, even if he must play the role oft he beffato, butt of the joke.« (Ebd.: 250)

Verspotten im Decamerone die Frauen die Männer, sind es in Ceis Text die kolonisierten »Indi«, die die Kolonisatoren verspotten. Cei schreibt, sie seien von den »Indi« verhöhnt (»beffati«, C:143) worden, indem sie Mais und essbare Wurzeln dem von den »Cristiani« so gesuchten Gold vorgezogen, »dicendo che non era buono da mangiare« (ebd.). Nach Hester wird in Ceis Text »the Indies-as-earthly-paradise trope« (Hester 2014: 245) zurückgewiesen, man könnte sogar sagen: demontiert.

Im Vergleich zu spanischen Texten zeigt sich das italienische koloniale Schreiben als unabhängiger von den Vorgaben des bürokratischen Apparats. Das Studium der italienischen kritischen Texte zu Ceis Relazione bringt andererseits zutage, dass mit ihnen eine mehr als unkritische und teilweise sogar explizite Heroisierung und Glorifizierung der >Entdeckung in den Forschungsdiskurs einfließt, als ob sie den Anschluss an die spanisch-, englischund deutschsprachige Kolonialforschung verpasst hätten.

\section{Der Brief des Juan Pérez de Tolosa an Kaiser Karl V. und die real cédula}

Der Brief von Juan Pérez de Tolosa an den König und die real cédula an den Indienrat werden aufeinander bezogen gelesen. Grundlage der Textanalysen ist José de Oviedo y Baños' ursprünglich 1723 erschienene Edition in der zweibändigen Fassung aus dem Jahr 1885 (vgl. P bzw. $\mathrm{K}^{45}$ ). Die Manuskripte liegen im Archivo Histórico Nacional in Madrid. Götz Simmer hat Tolosas Brief und das Mandat Karls V. ins Deutsche übertragen und in die Herausgabe der Textsammlung Tod am Tocuyo (vgl. Schmitt/Simmer 1999: 124-129 beziehungsweise 142-147) aufgenommen.

Juan Pérez de Tolosa wendet sich in seinem Schreiben vom 15. Oktober 1546, also knapp einen Monat nach der Vollstreckung seines Urteils gegen Juan de Carvajal, an den König. In seinem Brief führt Pérez de Tolosa Buch 
über sein Handeln gegen Carvajal. Im Modus der Rechtfertigung legt er besonderes Gewicht auf seine Prozessführung, um seine Dienste an den König vergütet zu bekommen. Damit bewegt sich das Schreiben im von Folger (vgl. 2011) beschriebenen Modus der »economy of mercedes«, dessen zentrale Funktion im Spanien der Frühen Neuzeit und seinen Kolonien kaum unterschätzt werden kann:

The discovery of America, the subjugation of its indigenous peoples, and its colonization provided the Spaniards with a huge number of those who sought to enrich themselves, striving for royal grants like encomiendas or offices. The principle pf the colonial economy of mercedes was to reward the beneméritos and their descendants, that is, the discoverers, conquerors and settlers (pobladores) who had served the Crown. (Ebd.: 22)

Diese »economy of mercedes« sei, so Folger (ebd.: 16), stark gesetzlich geregelt gewesen. Diese Regelungen ${ }^{46}$ hätten der Krone dazu gedient, die Etablierung von weitgehend unabhängigen lokalen Eliten in den neu eroberten Territorien zu verhindern und damit die Regierungsgewalt fest im Griff zu behalten. Die »economy of mercedes« ist untrennbar mit dem Problem der Distanzen verbunden, welche sich mit der Expansion eröffneten: »[T]he gulf separating overseas necessarily entailed a deferral of communication: A system of reports (relaciones, informaciones, memoriales) and written orders (cédulas, pragmáticas) supplanted the traditional governmental practice of verbal commands and supervision by superiors.« (Ebd.)

Das Machtmittel des Monarchen, Privilegien zu verwalten und an die Untertanen zu verteilen, so Folger weiter, sei schon früh bürokratisiert worden, habe sich jedoch im Zuge der kolonialen Expansion zu einer komplexen und hoch regulierten Prozedur entwickelt, die in den bekannten Relaciones de Méritos y Servicios, ${ }^{47}$ so etwas wie Anträge auf Entlohnung, ihren Höhepunkt gefunden habe (vgl. ebd.: 21). Die Gesetze legten Form, Inhalt und den Amtsweg fest, die bei der Einreichung der Gesuche eingehalten werden mussten. Nicht jedermann konnte einen Antrag stellen; nur gewisse Persönlichkeiten waren dazu berechtigt, die einen Zugang zum Zentrum der Macht besaßen (vgl. ebd.: 25).

Als Richter und Interimsgouverneur der Provinz Venezuela und damit Angehöriger des bürokratischen Apparats verfügte Pérez de Tolosa über eine solche Berechtigung, Antrag auf Vergütung zu stellen. Im Gegensatz zu seiner mehrdimensionalen Funktion als Richter, die er sowohl als kolonisiertes als auch kolonialisierendes Subjekt einnahm, hat die Interpellation in seinem 
Brief zwei Dimensionen in direkter Linie von ihm zum König. Zunächst ist sein Schreiben in ganz spezifischer Weise geprägt von der Distanz, die ihn von der Machtzentrale trennt. Tolosa leitet sein Schreiben ein, indem er seine »Zweifel« ausspricht, ob der König seine Nachricht (»despacho«) erhalten habe, weshalb er beschlossen habe, diese $\mathrm{zu}$ »duplizieren« (»duplicarlo en esta [carta]«, P: 219). Das Anfertigen von Kopien diente als Absicherung, die das Funktionieren der Kommunikation möglichst sicherstellen sollte. Das Wissen um das Risiko, dass der Kommunikationsfluss auf den weiten Wegen der Papiere unterbrochen werden könnte, ist vielen Texten eingeschrieben.

Ähnlich wie Ruiz de Vallejo stellt sich Tolosa als unverzichtbare, da einzige Informationsquelle der Geschehnisse in der Provinz dar, zu deren Eroberung und Verwaltung >die Deutschen (sprich: die Welser), mit Machtbefugnissen des Königs ausgestattet, gekommen seien (vgl. P: 219). Er möchte sicherstellen, dass nichts übrigbleibe, worüber sein Auftraggeber, der König, nicht informiert sei ("porque ninguna cosa quede de que V.A. no sea avisado«, ebd.). Er habe alle Informationen, die er anlässlich des Juicio de Residencia und auch bei anderer Gelegenheit zusammengetragen habe, noch einmal der Reihe nach (»uno en pos de otro«, ebd.) aufgeschrieben.

Auf diese einleitenden Worte folgt der Brief (vgl. Oviedo y Bañoz 1885: 220-224), ${ }^{48}$ der auf die Vergütung seines Dienstes am König und seiner damit zusammenhängenden Auslagen ausgerichtet ist. In seinem Schreiben rechtfertigt er sein Handeln lückenlos als notwendig; die Inhalte des Briefes richten sich ganz nach diesem Kriterium aus. Dazu gehört auch der Bericht über die Verhaftung Carvajals, womit Tolosa gleichzeitig die Lücke in den Akten des Prozesses schließt.

Pérez de Tolosa berichtet, wie er mit den Leuten, die er in Coro versammeln konnte, Carvajal gefolgt sei, um ihn zu verhaften. Sein Schreiben hält zunächst fest, dass er dem König bereits in einem früheren Brief von der Enthauptung vierer Hauptleute beziehungsweise Soldaten, angeordnet von Carvajal, berichtet habe (vgl. P: 220). Zwischen den Zeilen ist zu lesen, dass die Ungeheuerlichkeit dieser Tat das unmittelbare Einschreiten durch ihn, den Vertreter des Gesetzes, zwingend erforderte. Es folgt eine Erzählung, in der er auf seine Mühen, die er auf sich genommen und auf die Gefahren, denen er sich ausgesetzt hatte, fokussiert:

tomé el camino de las montañas pensando atajar camino, y sucedióme el camino muy trabajoso por la braveza de la tierra y de los indios de las montañas, que me dieron muchas guazabaras, yéndome siguiendo de noche y 
de día hasta pasar á los Ilanos que dicen de Carora; matáronme dos caballos y fueron heridos cinco cristianos, aunque ¡bendito sea Dios! ninguno peligró de muerte ${ }^{49}$ (P: 220).

Interessant erscheint hier das Bild der >Wildheit<, das wie schon bei Hutten sowohl für die Charakterisierung des Landes als auch der »indios« der Berge herangezogen wird.

Gemäß des Briefes ruhten sich die Männer nachdem sie 30 Tage marschiert waren, aus und erholten sich von den Strapazen in der Ebene von Carora, einem fruchtbaren und an Rotwild reichen Gebiet. Nach weiteren drei Tagen Marsch seien sie auf 17 berittene »cristianos « getroffen, die Carvajal ausgesandt habe, nachdem dieser von »indios« erfahren habe, dass ihn andere »cristianos« holen kämen. An dieser Stelle ergibt sich ein Widerspruch mit dem Bericht von Galeotto Cei, der ja gerade herausgestrichen hatte, dass Carvajal nicht auf die »indi« hören wollte: wiederum ein kleiner Hinweis darauf, dass Berichte von Augenzeugen keineswegs garantieren, die >Wahrheit< abzubilden. Pérez de Tolosa überwältigte seiner Darstellung nach die gegnerischen »cristianos « mit seiner Vorhut von 25 Berittenen und Fußsoldaten so geschickt, dass kein Einziger entkommen konnte. Nach dem Vorweisen seiner Ermächtigungsurkunden (provisones) hätten die Überwältigten gehorcht, und »como leales vasallos de V.A. $\aleph^{50}$ (P:220) hätten sie alles ausgeführt, was man ihnen befohlen habe. Diese Leute seien eine große Hilfe gewesen für das, was nachher geschehen sei (vgl. ebd.: 220f.). Die Botschaft: Pérez de Tolosa ist es gelungen, die Männer wieder in den sicheren Hafen der Loyalität zum König zu bringen. Damit hat er dem König einen großen Dienst erwiesen.

Dieses Muster wiederholt sich später im Text: Pérez de Tolosa, gewarnt von den Männern, dass sich Carvajal mit 60 Mann in den Savannen von Quibure aufhalte, beschloss, sich ohne Tross dorthin zu begeben und den Leuten die provisiones zu zeigen und sie so auf seine Seite $\mathrm{zu}$ bringen:

[C]aminé toda la noche, día de San Bartolomé, y en riendo el alba entré en este asiento, al cual había venido el mismo Carvajal á ver una amiga que tenía, y así lo tomé y prendí estando muy descuidado; y la gente del campo que con él estaba, estaba lo mismo, y aunque turbados, luégo vinieron a hacer la obediencia, especialmente un teniente suyo que se dice Juan de Villegas ${ }^{51}$ (P: 221).

Juan de Villegas wird dem König als sein treuer Gefolgsmann vorgestellt. Angeblich hatte Carvajal bereits die Nacht zuvor beschlossen, Villegas zusam- 
men mit sieben oder acht Männern zu enthaupten, weil er sich gegen seine Machenschaften gestellt habe. ${ }^{52}$ Villegas' Bild, das in den Akten des Prozesses beeinträchtigt worden war - Tolosa hatte ja sogar einen Haftbefehl gegen ihn ausgesprochen, und er wurde zu einer kleineren Geldbuße verurteilt -, musste >reingewaschen werden, denn er gehörte zu den Männern, die Tolosa nach dem Prozess für einflussreiche Posten in der Provinz vorsah. Tolosa sollte ihn ein Jahr darauf sogar zu seinem Stellvertreter und Generalkapitän ernennen, so dass Villegas - vor allem nach Tolosas Tod im Dezember 1548 - zur höchsten Autorität der Provinz aufstieg (vgl. Simmer 1999b: 188). Die geschilderten Verhältnisse gestatten es Pérez de Tolosa, sich selbst als Retter aus der ausweglosen Situation darzustellen: »[T]iénese por averiguado que si no llegara yo á la sazón, que todos se hicieran pedazos cuantos estaban en este campo ${ }^{53}$ (P: 221). Am Schluss vereinigten sich alle, wie im letzten Aufzug eines Dramas, den provisiones gehorchend, und »todos con mucha alegría vinieron á dar la obediencia ${ }^{54}$ (P: 221). Sein Plan, wie die Provinz gerettet werden sollte, zu dem auch der Einsatz Villegas' gehörte, musste sorgfältig diskursiv vorbereitet und gerechtfertigt werden, um ihn in die Tat umsetzen zu können. Dazu stellte er die Provinz Venezuela als »zerrüttet« (Übersetzung Simmer, Schmitt/Simmer 1999: 127) dar (vgl. P: 223); die Bewohner seien so arm und heruntergekommen, dass er für Coro keine Zukunft sehe. Er habe seinen Bruder mit 40 Reitern und 60 Landsknechten zur Erkundung eines Tals geschickt. Falls sie dort Minen fänden, könnte das die Rettung der Provinz sein. Er selbst bleibe mit 70 Mann in dem von sehr armen »indios« dicht besiedelten Ort (El Tocuyo). An dieser Stelle - gegen Ende des Schreibens - wird klar, wozu diese Beschreibung der prekären Verhältnisse (auch) dient: Da er seine Entlohnung aufgrund des Mangels in der Provinz gefährdet sieht, möchte er die Krone veranlassen, dass man ihn in Santo Domingo statt in der Provinz Venezuela entschädigt. Seine Majestät habe Anweisung gegeben, falls es hier (das heißt in der Provinz Venezuela) an Ressourcen fehle, mit denen Pérez de Tolosas Salär gedeckt werden könne, solle man ihn in Santo Domingo bezahlen. Und diesen Fall sieht der Richter nun als gegeben, denn der Provinz fehlt es seinen Angaben nach an Gold (vgl. ebd.: 224). Seiner Position offenbar bewusst, verhandelt er offen über die Höhe seines Salärs:

V. A. me hizo merced de mandar que si aquí no hubiese de qué se me pagase mi salario, se me pagase en Santo Domingo, y porque aquí no hay aparejo para se me pagar, porque, como he dicho, ningund oro hay, suplico á Vuestra Alteza sea servido mandar en Santo Domingo se me pague el salario, en bue- 
na moneda y á respeto de lo que vale en Sevilla, porque de otra manera yo recibiría gran pérdida y el salario no respondería con la tercia parte al salario que V.A. fué servido que se me pagase en esta provincia, que es seiscientos y cincuenta mil maravedís por año, y segund la costa, yo debería en Santo Domingo, á personas que me proveen, más de lo que monta el salario ${ }^{55}$ ( $P$ : 223f.).

Pérez de Tolosa unterstreicht in seinem Brief an mehreren Stellen, dass er selber den durch und durch boshaften Übeltäter Carvajal aus dem Verkehr gezogen habe. Carvajals Missetaten machten seinen eigenen $\mathrm{Zug}$ zu seiner Verhaftung unentbehrlich, womit Tolosa gleichzeitig seinen Anspruch auf Bezahlung unterstreicht: "Asimismo á V. A. suplico mande tener respeto á los trabajos y costa que me ha sucedido en tener necesidad de salir de Coro, en tan largo camino y al servicio que en ello se ha hecho, y mande que se me dé la ayuda de costa que V. A. fuere servido ${ }^{56}$ (P: 224).

Indem er die Abschrift der Geständnisse von Carvajal und des Urteilsspruchs beilegt, liefert er die Rechtfertigung für sein Handeln als Richter, nämlich dass er Carvajal rechtmäßig angehört und verurteilt habe. Weiter berichtet Pérez de Tolosa, dass Carvajals Hab und Gut konfisziert worden sei; es habe aber seine Schulden nicht überstiegen. Es habe zwar noch andere Verurteilungen gegeben, jedoch zu wenige, um Carvajals Schulden und die Kosten des Verfolgungszugs zu seiner Verhaftung zu decken. Indirekt wird damit an den König appelliert, ihm seine auf eigene Kosten durchgeführte Verfolgungsjagd zu vergüten. In einem Nebensatz wird überdies eine Lücke im Informationsfluss sichtbar, die einer ganz profanen praktischen Ursache geschuldet ist, dem Papiermangel in der Provinz: Von den Verurteilungen weiterer Personen »no se envía relación por falta de papel ${ }^{57}$ (P: 224).

Den Welsern und ihren Gouverneuren weist Juan Pérez de Tolosa klar die Schuld zu, mit ihrer Nichterfüllung des Kronvertrags zur Verarmung der Siedler beigetragen zu haben. Denn weil sie keine Dörfer gegründet hätten, habe den Siedlern die Möglichkeit gefehlt, sich eine Lebensgrundlage zu schaffen. An all dem »los Gobernadores tuvieron la culpa, porque mejor se pudieron decir destruídores y despobladores de indios, al sacar de las perlas, que basta agotar las Indias de gente ${ }^{58}$ (P: 222). In der Anspielung auf die wenige Jahre zuvor erlassenen Leyes Nuevas (siehe Kapitel 1) zeigt sich der Schreibende als loyal und im Einklang mit den Anweisungen des Hofes, auch wenn ihm vorher die »indios« niemals explizit ein Anliegen waren. Die Männer der Truppe von Carvajal träfe keine Schuld an der Misere. Im Gegenteil: 
Allesamt seien sie "gente muy lucida y muy diestra en las cosas de las Indias y de mucha experiencia de guerra ${ }^{59}$ (P: 222), und alle strebten danach, ihrer Majestät zu dienen (vgl. ebd.). Dass sie sich noch nicht gegen Carvajal erhoben hätten, liege daran, dass dieser ihnen Hoffnung gemacht habe, sie nach Neu Granada zu bringen.

Pérez de Tolosa schildert das Handeln und Wirken der Welser in der Provinz Venezuela in den dunkelsten Farben. In dieser Hinsicht sind die Texte nicht einheitlich; sie geben auch andere Ansichten zu lesen. Beispielsweise sagte Pedro de San Martín im Juicio de Residencia zu den Welsern am 20. Oktober 1547 aus, dass die Spanier, die in jene Provinz gekommen seien, kein Gold gehabt hätten, um sich auch nur das Allernötigste zu besorgen. Die Welser hätten ihnen die Waren jedoch zu sehr gerechten und moderaten Preisen abgegeben. Das Gold, das von den entradas und von Maracaibo zusammengetragen worden sei, sei gerecht verteilt worden, und die Bewohner hätten mit der einen Hälfte des Geldes ihre Einkäufe frei tätigen können, als Schiffe in Coro angelegt und Waren zum Verkauf angeboten hätten. Mit der anderen Hälfte sei ein Teil der Schulden bei den Welsern getilgt worden (vgl. ebd.: 226). Dass Pérez de Tolosas Schreiben dagegen eine dezidiert kritische Position zu den Welsern einnimmt, lässt sich (auch) mit seiner Interessenlage erklären: Um angemessen entschädigt zu werden, muss er sein Wirken in der Provinz als erfolgreich abgeschlossen darstellen; dem König musste klar gemacht werden, dass die Provinz aufgrund der Misswirtschaft der Welser am Abgrund gestanden hatte und dass er, Pérez de Tolosa, sie auf den Weg zum Erfolg bringen konnte.

Dass er den Antrag auf Vergütung seiner Dienste in Form eines Briefes abgefasst hatte, entsprach den Vorgaben des bürokratischen Apparats. Es ist meines Wissens nicht bekannt, ob eine Einschätzung der Anträge beziehungsweise seine Entschädigung stattgefunden hat. Der Ansatz einer Antwort deutete sich jedoch wenig später auf einem anderen Parkett an: In einer real cédula vom 11 . Februar 1548 wies Karl V. den Indienrat an, das Verfahren zu den Enthauptungen Huttens und Welsers zu Ende zu führen, da Richter Tolosa Juan de Carvajal zwar überführt, alle anderen Schuldigen jedoch nicht bestraft habe. Damit kann dieses kaiserlich-königliche Schreiben als eine Art Antwort auf Tolosas Brief gelesen werden, denn es nimmt Bezug auf Tolosas Handeln als Richter im Kriminalprozess gegen Juan de Carvajal, indem es feststellt, dass dieser nicht vollständig - das heißt nicht gegen alle Schuldigen - Gerechtigkeit geübt habe. Mit keinem Wort geht das Schreiben jedoch auf Pérez de Tolosas Brief ein, in dem dieser begründet hatte, warum er Car- 
vajal als Einzigen verurteilte. Damals hatte er argumentiert, dass Ruhe in der Provinz einkehren müsse:

Carvajal fué oído ordinariamente, y concluído el pleito fué sentenciado y en él ejecutada la sentencia, segund parece por el traslado sinado [wohl: signado] de sus confisiones [wohl: confesiones] y sentencia, que con ésta envío: otra sentencia corporal no la hubo ni se ejecutó, porque solo él bastaba para inficionar más gente de la que aquí estaba: por el bien y concordia desta gente no convino al servicio de V. M. que más se hiciese, y con lo hecho no hay gente de más unión y conformidad, para lo que toca al servicio de V. M., de la que aquí está60 (P: 223).

Warum ging Karl V. in seinem Mandat mit keinem Wort auf diese Argumentation ein? Hatte der König Tolosas Brief überhaupt bekommen, und war er informiert über dessen Inhalt? Wie ist der Umstand zu deuten, dass die beiden - Tolosa und der König - zwar von ein und derselben Situation sprechen und damit im Grunde genommen Kommunikationspartner wären, die von Tolosa eingeleitete Kommunikation aber nicht aufgenommen und beantwortet wurde? Der durch diese Fragen umrissene Umstand ist bemerkenswert, hatte der König doch Tolosa immerhin direkt mit dem Auftrag betraut, als Richter und Gouverneur auf Zeit in der Provinz Venezuela zu walten.

Das Mandat Karls V. nimmt also keinen direkten Bezug auf Pérez de Tolosas Schreiben; es bezieht sich dagegen explizit auf Informationen, die dem König von den Hinterbliebenen der beiden Opfer Hutten und Carvajal zugetragen worden waren. Gemäß des bei Schmitt/Simmer übersetzten Mandats Karls V. vom 11. Februar 1548 hatten Philipps älterer Bruder, Moritz von Hutten, und Bartholomäus der Ältere in zwei Supplikationen an den Kaiser »die Strafverfolgung sämtlicher Mittäter Carvajals sowie die Herausgabe der Habe der Ermordeten «(Schmitt/Simmer 1999: 143) gefordert. Das königlich-kaiserliche Mandat nahm offenbar »wörtlich den Text ihrer Eingaben « auf. Auch die Idee, dass die beiden Opfer »erhebliche Werte an Gold, Silber und Edelsteinen bei sich gehabt« und »über wichtiges Wissen verfügt hätten« (ebd.), stammte von den Supplikanten. Sogar deren Unterstellung, der Besitz dieser Dinge sei Carvajals Motiv für die Enthauptungen gewesen, übernahm das kaiserliche Schreiben, das damit den Familien der Hutten und Welser Recht zu geben scheint.

Tatsächlich aber stieß, so Schmitts Einschätzung, das Mandat ins Leere (vgl. ebd.) und blieb »ohne Folgen« (ebd.). Diese Aussage greift jedoch zu kurz; man bedenke, dass es angesichts des ausgeklügelten bürokratischen Apparats 
kaum vorstellbar ist, dass der König (oder einer seiner Magistraten) zur Feder greift und eine real cédula herausgibt, nur um sins Leere zu stoßen<. Auch die Tatsache, dass es damals »in allen politischen Kanzleien üblich«war, dass die Supplikationen wörtlich übernommen wurden und damit "ganz ihrer Sichtweise und Argumentation « (ebd.) folgten, kann auf einen strategisch ausgerichteten Charakter dieser kanzleimäßigen Praxis hindeuten. So könnte sie als Modus der Interpellation gedeutet werden; das Mandat würde dann vorführen, wie die Interpellation durch den Träger der Macht in Gang gesetzt werden konnte. Das strategische Ziel könnte darin bestehen, die Supplikanten über den Kanal der Interpellation in den bürokratischen Apparat einzubinden, indem das Schreiben die Worte der Eingaben und damit die Anliegen in einer für die Supplikanten erkennbaren Weise aufnimmt und sie gewissermaßen mindestens teilweise ruhigstellt.

Welche der gegebenenfalls konkurrierenden Supplikationen in die Schreiben aufgenommen wurden, muss dann von der Interessenlage der Beteiligten abhängig gewesen sein. Damit ein Supplikant sein Ziel bei der Krone erreichte, mussten seine Papiere nicht nur das Tor zum Hof passieren, sondern er musste in seinem Schreiben Informationen liefern, welche die Wirkung anderer, eventuell rivalisierender Informationen gewissermaßen ausschalteten. Nur so konnte sichergestellt werden, dass die Kommunikation in die gewünschte Richtung gelenkt wurde. Im vorliegenden Fall hatten offenbar die Supplikationen der Hutten und Welser die Oberhand gegen das Schreiben von Juan Pérez de Tolosa gewonnen, aus welchen Gründen auch immer.

Ohne Folgen in und für die Textwelt bleibt immerhin, dass sich die real cédula wie ein kleines Stück Heldenepos über Philipp von Hutten liest, indem dieser als Entdecker »viele[r] sehr reiche[r] Länder und Provinzen« modelliert wird:

Por parte del Rdo. Obispo de Aystat y Guillermo de Huten, su Hermano, y Bartolomé y Antonio Belzeres, nos ha sido hecha relación que Phelipe de Huten, hermano del dicho Obispo y Guillermo, pasó con licencia nuestra á la Provincia de Venezuela y Cabo de la Vela en el armada que llevó el gobernador Jorge Hohermut etc. Habiendo Ilegado y desembarcado en la dicha provincia el dicho Gobernador, fué á un descubrimiento adonde murió, y por su fin y muerte la Audiencia que reside en la isla de Santo Domingo proveyó por capitán general de la dicha provincia al dicho Philippe de Huten, el cual entró la tierra adentro, en que se ocupó cinco años y descubrió muchas tierras y provincias muy ricas etc. Volviendo el dicho Philippe el año pasado 
de 46 á dar noticia de su descubrimiento, halló que el año antes los oidores de la dicha Audiencia, sin saber que fuese vivo ó muerto, ni tener comisión ni nombramiento de los dichos Belzeres, habían proveído por gobernador y capitán general de la dicha provincia á un Juan de Carvajal, el cual, sabido que el dicho Fhilipe [sic!] de Huten venía rico y había descubierto tierras y provincias ricas, con desordenada codicia y maldad prendió al dicho Phelipe [sic!] de Huten, y en su compañía á Bartlomé Belzer el mozo y á Alonso Romero y Gregorio de Plasencia, á todos los cuales, sin causa alguna, el dicho Juan de Carvajal los degolló por tomar y robar lo que el dicho Phelipe de Hutten traía, é por gozar él del dicho descubrimiento ${ }^{61}$ (K: 277f.).

Dass Carvajal aus niederen Beweggründen gehandelt habe, wurde bekanntlich bereits in den Akten des Prozesses festgestellt und ist nichts Neues; dass er dagegen Hutten seine Besitztümer »rauben« wollte und aus Habgier gehandelt haben soll, widerspricht den Ergebnissen des Prozesses: Gemäß den Akten war bei Carvajal nichts gefunden worden, was man den Familienangehörigen der Opfer hätte weitergeben müssen. Mit anderen Worten: Das Mandat Karls V. liefert eine andere Version der Ereignisse um die Enthauptungen als die Akten des von Pérez de Tolosa geführten Prozesses. Darüber, was Eingang findet in Geschriebenes, entscheidet offenbar das Gefüge der Interessen, die den Schreibenden in Form von Informationen erreichen. In diesem Fall bedient das Schreiben des Königs die Interessen der Supplikanten, der Familie Hutten und Welser. Pérez de Tolosa scheint abgeschlagen; die in seinem Brief zur Schau gestellten Bemühungen werden nur ansatzweise aufgenommen. Dem Kaiser wurde berichtet,

que aunque el Licenciado Tolosa, juez de residencia de la dicha provincia, hizo pesquisa é información del dicho delito, y averiguada la verdad arrastró y ahorcó al dicho Juan de Carvajal, dando por leales vasallos y servidores nuestros á los dichos Phelipe de Huten y Bartolomé Belzer el mozo, no hizo justicia de los otros que fueron en consejo y hecho de la dicha maldad y traición que cometió, ni mandó restituir los bienes que quedaron del dicho Phelipe de Huten y Bartolomé Belzer el mozo, y los otros dos que con ellos murieron, á sus herederos, debiéndolo hacer, suplicándonos mandásemos que demás del dicho Juan de Carvajal se hiciese rigurosa justicia contra todas los que fueron en dicho hecho y consejo de la muerte de las sobredichas personas, é que todos aquellos que se hallaren cuplantes en ella se traigan presos y á buen recaudo á estos reinos para que vosotros hagáis justicia en su casua conforme á derecho, pra que á ellos sea castigo y á otros ejemplo; 
é que también se mandase al dicho Licenciado Tolosa y á otras cualesquier cosas que á los dichos Phelipe de Huten, Bartolomé Belzer el mozo, Alonso Romero y Gregorio de Plasencia pertenecían en cualquier manera, así de sus repartimientos como de otras cosas, liquidando y averiguando la verdad, lo envíen luego á buen recaudo, con realción cierta y cercadera de todo ello, á los nuestros oficiales de la cas de la contratación que residen en la ciudad de Sevilla, para que se acuda con ello á quien de derecho lo hubiere de haber ${ }^{62}$ (K: 277f.).

Diese Version der Geschichte um den Fall Hutten-Carvajal wäre eigentlich die Trivialste und Eingängigste. Trotzdem hat sie es bis heute nicht geschafft, die anderen zu verdrängen, obwohl sie aus dem Munde des Mächtigsten aller Mächtigen stammte. 